Supporting Information:

\title{
Chemically Exfoliated SnSe Nanosheets and Their SnSe/Poly(3,4- ethylenedioxythiophene):Poly(styrenesulfonate) Composite Films for Polymer Based Thermoelectric Applications
}

\author{
Hyun Ju and Jooheon Kim* \\ School of Chemical Engineering \& Materials Science, \\ Chung-Ang University, Seoul 156-756, Republic of Korea
}

*Corresponding author: jooheonkim@cau.ac.kr (J. Kim)

Supporting Information Contents:

1. Figures

2. Tables

3. Experimental procedures 


\section{Figures}
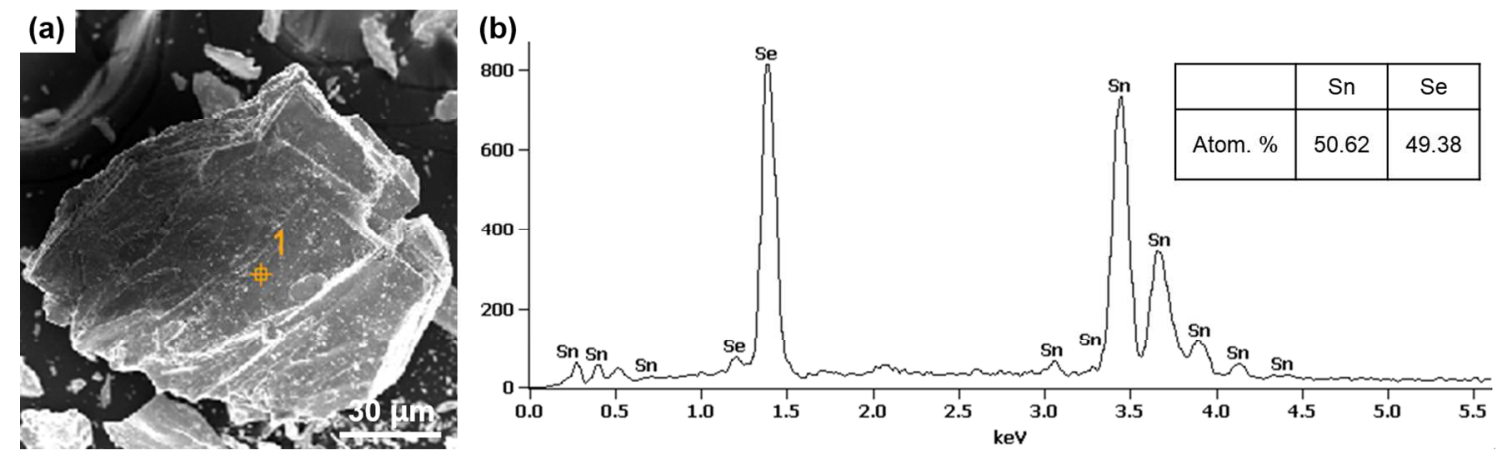

Figure S1. (a) FE-SEM image for the prepared SnSe particle and (b) the corresponding EDS spectrum.
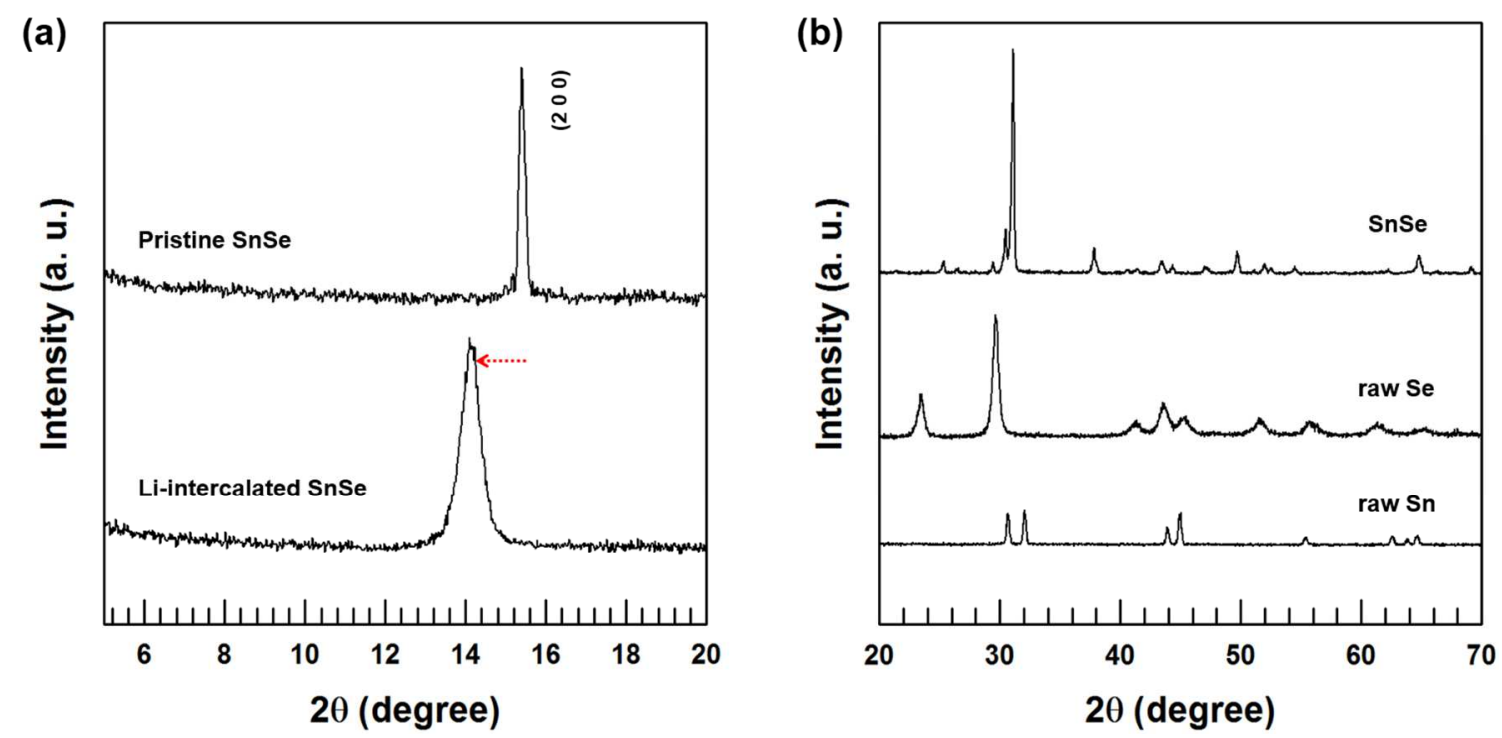

Figure S2. XRD patterns of (a) pristine SnSe powder and Li-intercalated SnSe, and (b) the raw Sn, raw Se, and the synthesized SnSe NSs. 


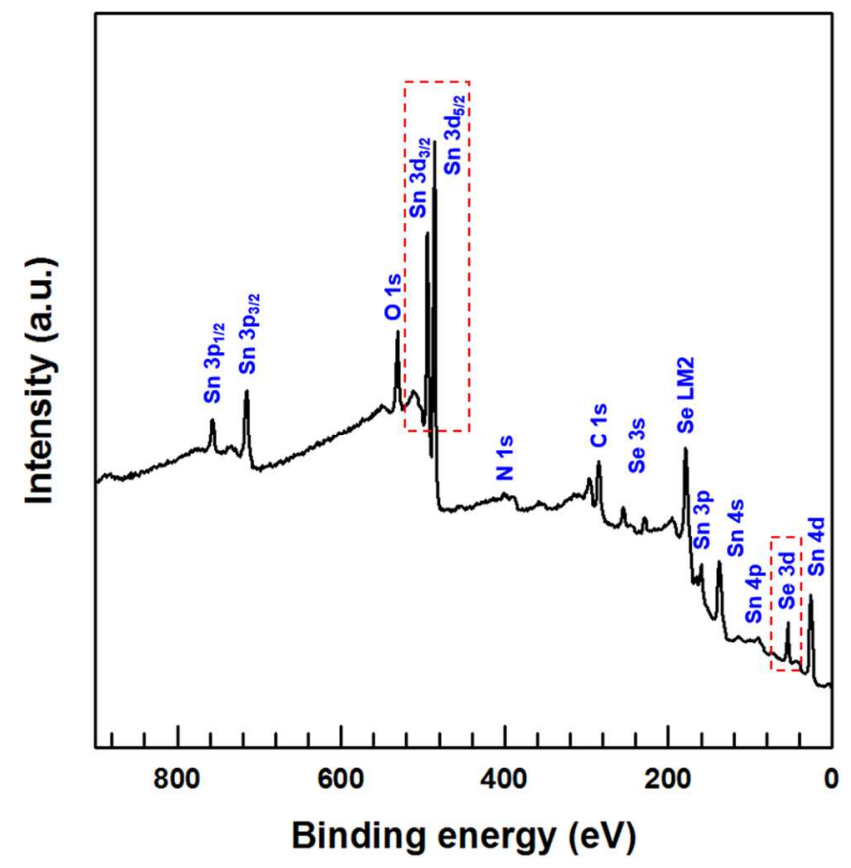

Figure S3. XPS survey spectrum of the as-prepared SnSe NSs. The red dotted prisms outline Sn $3 \mathrm{~d}$ and Se $3 \mathrm{~d}$ core levels.

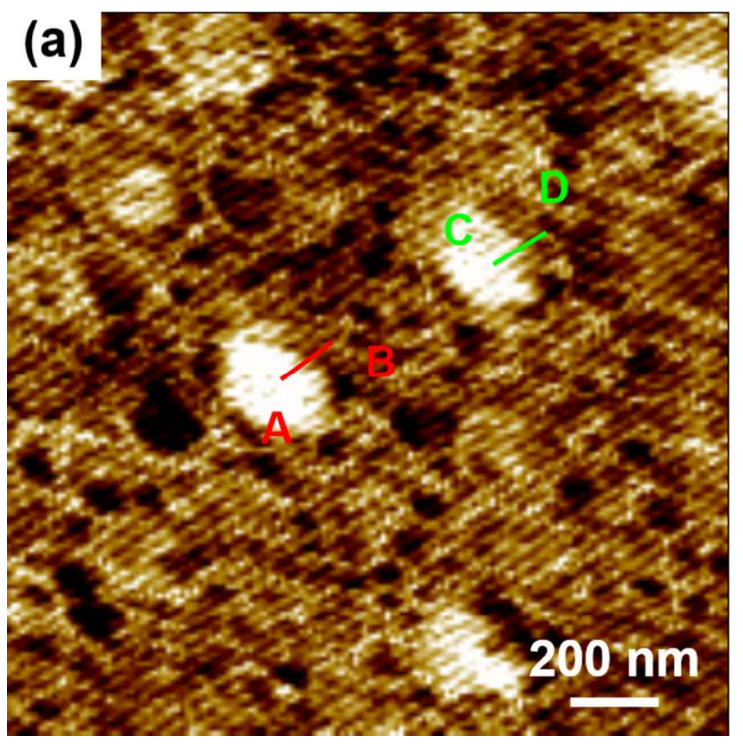

(b)
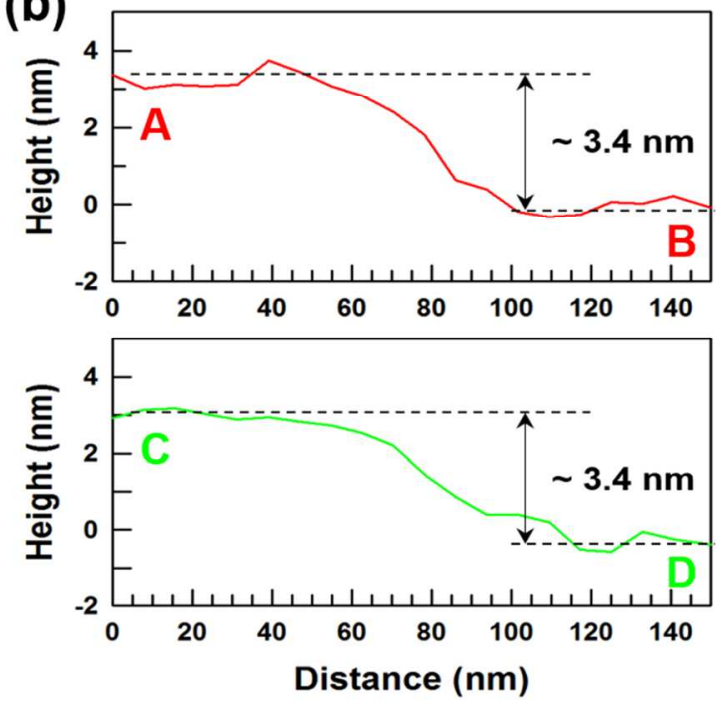
Figure S4. (a) AFM image of the exfoliated SnSe nanosheets and (b) the corresponding height profile.
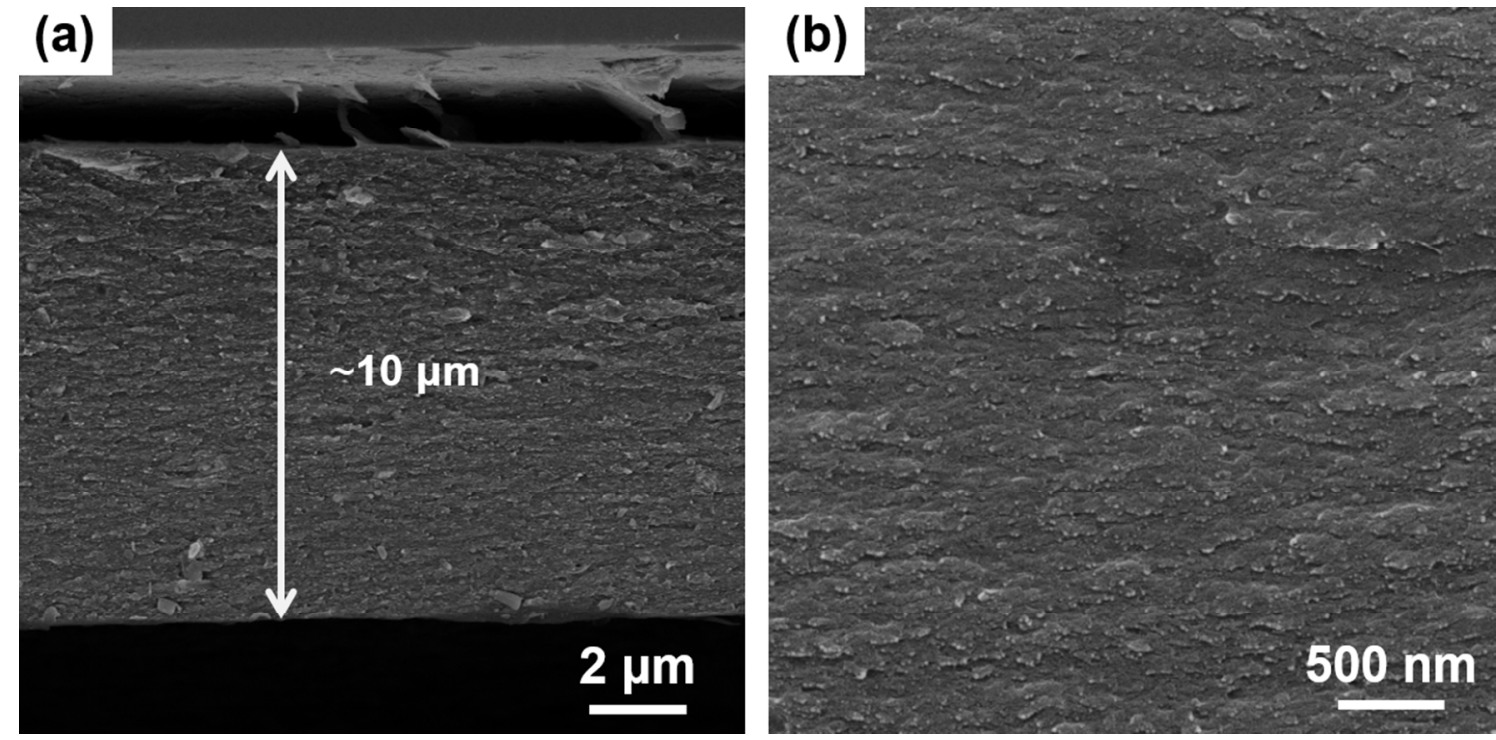

Figure S5. (a) Low- and (b) high-resolution cross-sectional FE-SEM images of drop-cast SnSe/PEDOT:PSS film with SnSe content of 20 wt.\%. 

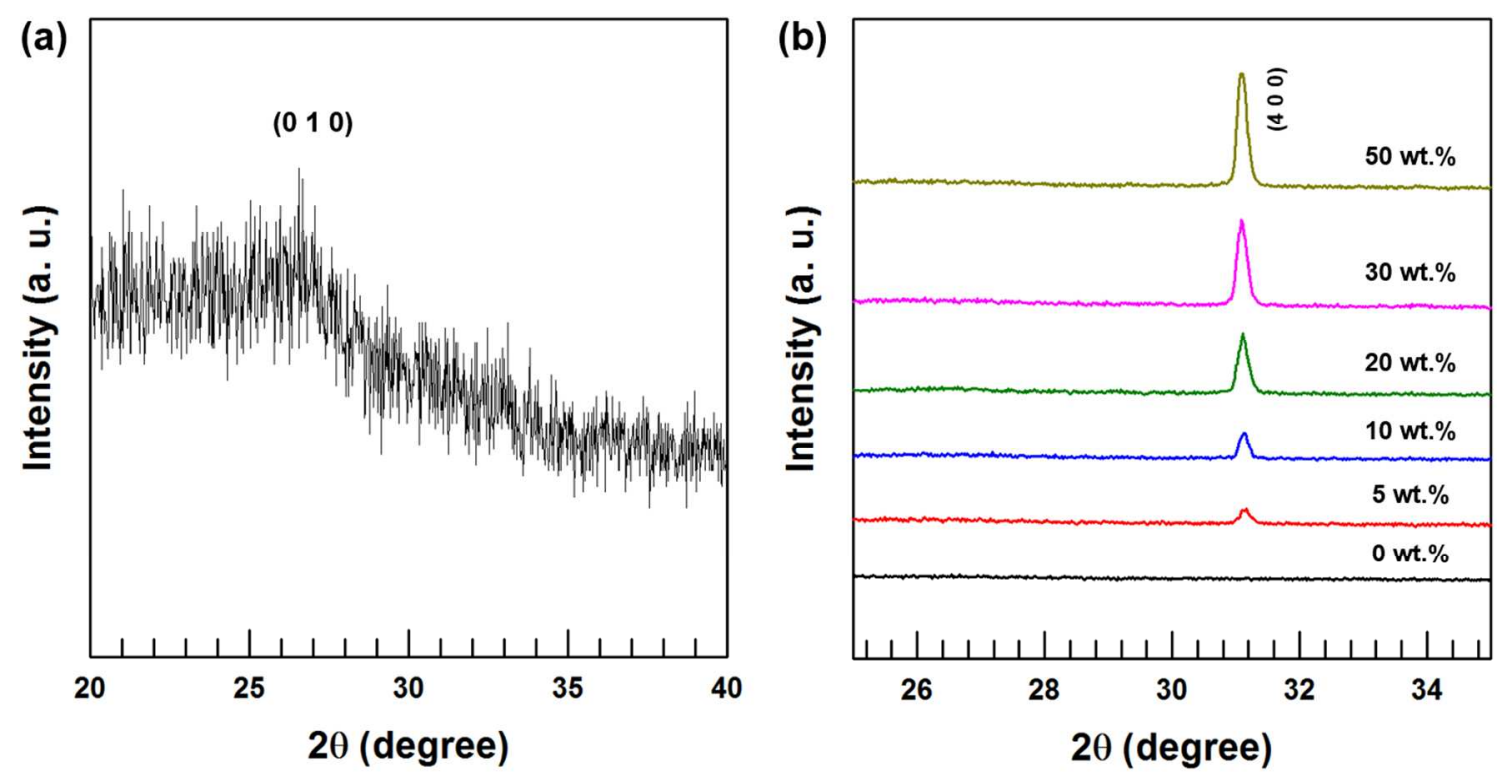

Figure S6. XRD patterns of (a) the pristine PEDOT:PSS and (b) the SnSe/PEDOT:PSS composites with varying SnSe content.
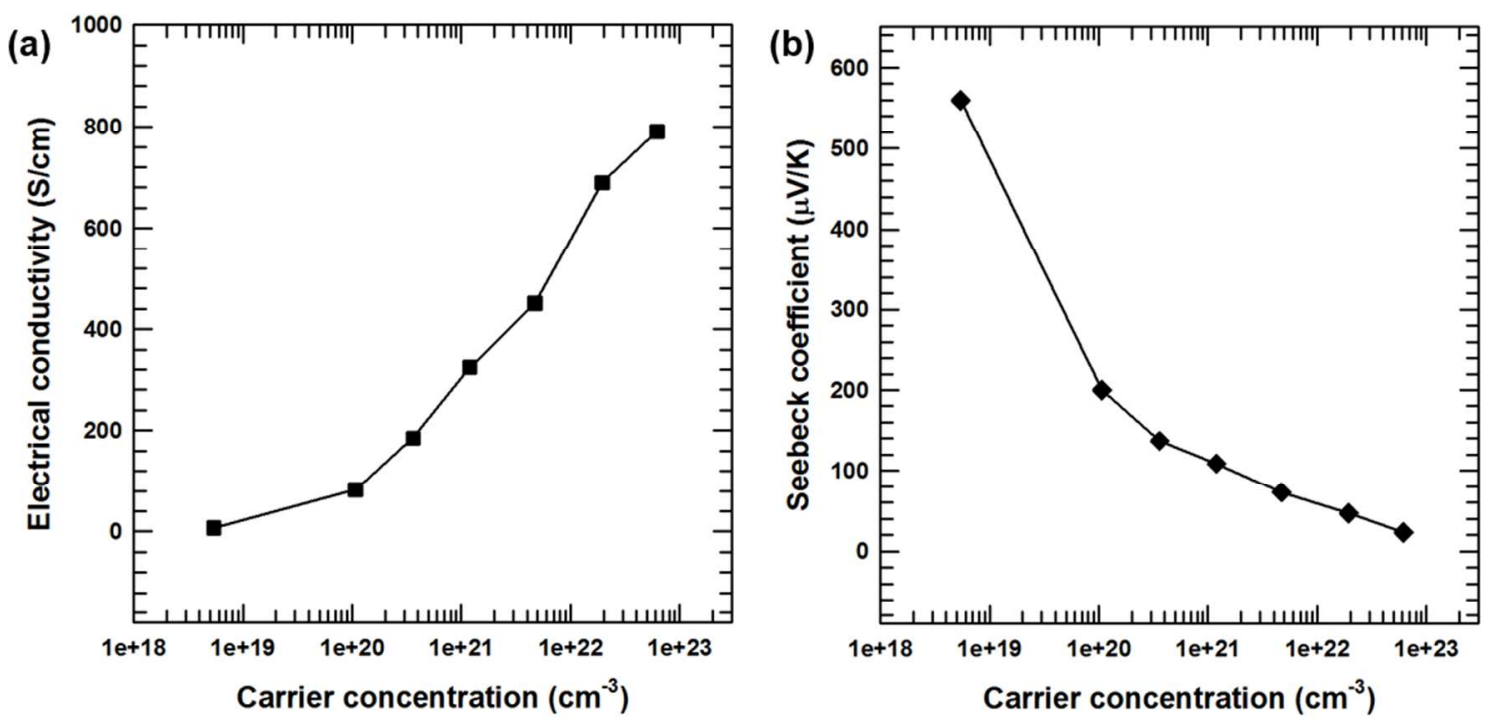

Figure S7. (a) Electrical conductivity and (b) Seebeck coefficient of the prepared SnSe NS/PEDOT:PSS composites as a function of carrier concentration. 

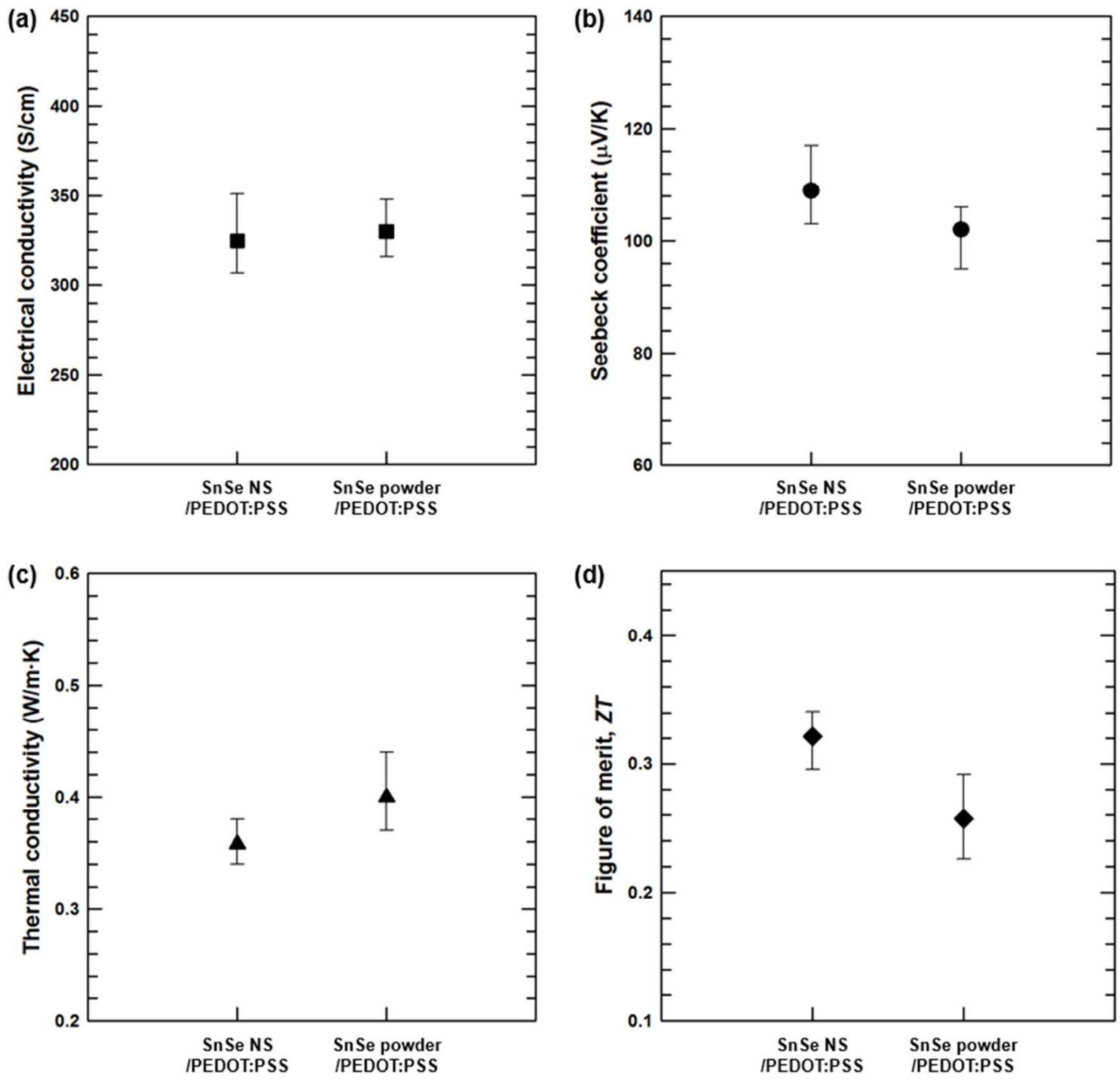

Figure S8. (a) Electrical conductivity, (b) Seebeck coefficient, (c) thermal conductivity, and (d) $Z T$ values for the SnSe NS/PEDOT:PSS and SnSe powder/PEDOT:PSS composites with SnSe contents of $20 \mathrm{wt} . \%$.

\section{Tables}




\begin{tabular}{ccccc}
\hline & $\alpha$ & $\rho$ & $C_{P}$ & $\kappa$ \\
& $\left(\mathrm{mm}^{2} / \mathrm{s}\right)$ & $\left(\mathrm{g} / \mathrm{cm}^{3}\right)$ & $(\mathrm{J} / \mathrm{g} \cdot \mathrm{K})$ & $(\mathrm{W} / \mathrm{m} \cdot \mathrm{K})$ \\
\hline PEDOT:PSS & 0.160 & 1.301 & 1.210 & 0.25 \\
SnSe (5) & 0.181 & 1.508 & 1.032 & 0.28 \\
SnSe (10) & 0.199 & 1.726 & 0.908 & 0.31 \\
SnSe (20) & 0.218 & 2.088 & 0.786 & 0.36 \\
SnSe (30) & 0.274 & 2.572 & 0.570 & 0.40 \\
SnSe (50) & 0.304 & 3.343 & 0.432 & 0.44 \\
\hline
\end{tabular}

Table S1. Thermal properties of SnSe/PEDOT:PSS composites with various SnSe contents. SnSe

(x) indicates the $\mathrm{x}$ weight percent ratio of SnSe nanosheets $(5,10,20,30$, and $50 \mathrm{wt} . \%)$.

\section{Experimental procedures}


3.1 Fabrication and thermoelectric characterization of pristine SnSe NS sample

The SnSe nanosheets were pressed into a thin pellet for $10 \mathrm{~min}$ at $823 \mathrm{~K}$ under $50 \mathrm{MPa}$. Then, a square sample with $10 \mathrm{~mm} \times 10 \mathrm{~mm} \times 1 \mathrm{~mm}$ dimensions was cut from the pellet and used to measure the electrical conductivity and Seebeck coefficient. The thermal conductivity was investigated using a disk-shaped sample from the pellet. Five samples were prepared to demonstrate the reproducibility of our experiment. The characterization equipment was the same as that used for the SnSe/PEDOT:PSS composite samples. 